\title{
Calcium homeostasis in women with non-metastatic breast cancer with osteoporosis after a single dose of denosumab: a pilot study
}

Unit of Endocrinology, Theagenio Cancer Hospital of Thessaloniki

Stylianos Mandanas, Konstantinos Toulis, Lemonia Mathiopoulou, Efterpi

Margaritidou, Konstantinos Georgopoulos, Maria Boudina, Alexandra Chrisoulidou, Kalliopi Pazaitou-Panayiotou

Aim: To assess the calcium homeostasis after treatment with denosumab in women with non metastatic breast cancer

Patients and Methods: We prospectively enrolled 42 female patients with osteoporosis, who were eligible for treatment with denosumab. Patients were divided in two groups. In Group A were included 24 healthy postmenopausal women (controls) and in group B were included 18 patients with non metastatic breast cancer.

A single-dose of denosumab (60mg) was administered to both groups, under standard calcium and vitamin D supplementation. Serum calcium, phosphorus, parathyroid hormone (iPTH) and 24hr urine calcium were measured at days 0,7 and 180 . Primary outcomes were the development of hypocalcaemia and secondary hyperparathyroidism.

Results: At baseline, both groups were comparable in age, calcium and iPTH levels. No events of hypocalcaemia were recorded. Overall, incidence of secondary hyperparathyroidism was found to be $45.5 \%$ one week after administration of denosumab At day 7, iPTH was found to be significantly higher only in controls (Wilcoxon Signed Rank test: $p=$ 0.013) compared to group-specific baseline values. Interestingly, at day 180 incidence of secondary hyperparathyroidism was higher in Group B in contrast to the pattern recorded in controls, although not reaching statistical significance. At day 180, borderline increase in iPTH of Group B was noted ( $p$ $=0.08$ ), whereas $\mathrm{iPTH}$ returned to baseline in controls.

Conclusions: A pattern of delayed development of secondary hyperparathyroidism might be present in patients with non-metastatic breast cancer. It could be extrapolated that this finding might be associated with a partial functional defect in calcium sensing receptor, which has recently been implicated in the pathogenesis of breast cancer. The findings warrant further investigation.

\begin{tabular}{ll|ll} 
& & Group A & Group B \\
\hline Age & & 59.5 & 57.5 \\
\hline Ca & 0 & 9.79 & 10.015 \\
& 7 & 9.285 & 9.655 \\
& 180 & 9.63 & 9.72 \\
\hline P & 0 & 3.57 & 3.855 \\
& 7 & 3.085 & 3.6 \\
& 180 & 3.325 & 3.61 \\
\hline PIHI & 0 & 42.995 & 46.53 \\
& 7 & 68.55 & 50.2 \\
& 180 & 45.64 & 60 \\
\hline
\end{tabular}

Table: Biochemical changes from day 0 to 180 , Ca: calcium, P: phosphorus, PTH: parathyroid hormone, 0: at baseline, 7: day 7, 180: day 180 . Data are median 